\title{
Development of Large Area Hybrid Photodiodes for the LHCb Ring Imaging Cherenkov Detectors
}

A. Braem ${ }^{a}$, E. Chesi ${ }^{a}$, W. Dulinski ${ }^{b}$, F. Filthaut ${ }^{a}$, A. Go ${ }^{a}$, C. Joram ${ }^{a, 1}$, G. Lion ${ }^{a}$, J. Seguinot ${ }^{c}$, P. Weilhammer ${ }^{a}$, P. Wicht ${ }^{a}$ and T. Ypsilantis ${ }^{d}$

${ }^{a} \mathrm{CERN}$, Switzerland

${ }^{b}$ LEPSI, Strasbourg, France

${ }^{c}$ College de France, Paris

${ }^{d}$ INFN Bologna, Italy

${ }^{1}$ corresponding author 


\begin{abstract}
We report on the development of large area hybrid photo diodes (HPD) which are one of the proposed photodetectors for the RICH counters of the LHCb experiment. The HPD's consist of a cylindrical vacuum envelope of $127 \mathrm{~mm}$ diameter capped with a spherical borosilicate UV-glass entrance window. Focusing electrodes demagnify the image on a silicon detector of $50 \mathrm{~mm}$ diameter comprising 2048 pads with a surface of $1 \mathrm{~mm}^{2}$ each. The analogue readout electronics is integrated in the vaccum tube. As an intermediate step a HPD with a UV sensitive CsI photocathode has been produced which allowed to verify the electron optics of the HPD. A large UHV evaporation plant for the production of HPD's with visible light transmittive bialkali photocathodes $\left(\mathrm{K}_{2} \mathrm{CsSb}\right)$ has been built and successfully operated. The evaporation process is optimized for maximum quantum efficiency and life time of the photocathodes. A cold Indium sealing technique developed for a minimum thermal load of the photocathode and the silicon sensor has proven to provide excellent vacuum tightness.
\end{abstract}




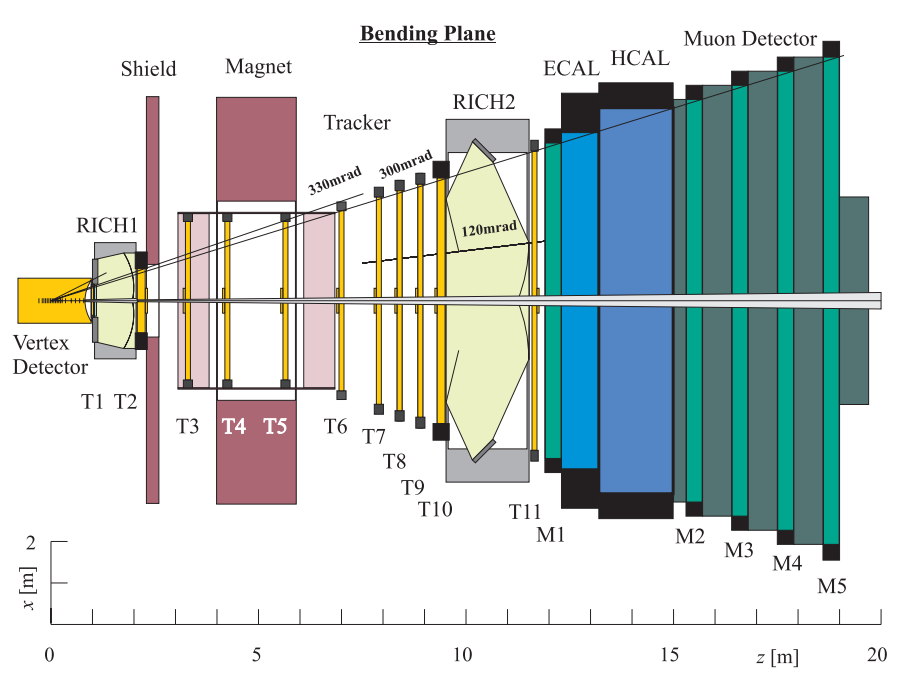

Figure 1: Outline of the LHCb spectrometer. The two RICH detectors are positioned behind the microvertex detector (RICH 1) and in front of the electromagnetic calorimeter ( $\mathrm{RICH} 2)$.

\section{Introduction}

The single arm spectrometer LHCb is designed for CP asymmetry and rare decay studies in the system of B-mesons. LHCb will profit from the high $b \bar{b}$ cross section and luminosity at the LHC collider. The outline of the recently approved LHCb spectrometer [1] is shown in Fig. 1. It includes two Ring Imaging CHerenkov (RICH) detectors for $\pi / \mathrm{K}$ separation in the momentum range $1-150 \mathrm{GeV} / \mathrm{c}$. The Cherenkov light emitted from the various radiators is focused by means of spherical mirrors onto detection planes outside the acceptance of the $\mathrm{LHCb}$ spectrometer. In total a surface of $2.9 \mathrm{~m}^{2}$ has to be equipped with photodetectors which have to provide a granularity of about $2.5 \times 2.5 \mathrm{~mm}^{2}$. Hexagonal close packing of round photodetectors with an active surface coverage of $80 \%$ results in a total number of electronic channels of about 340,000 .

A comprehensive R\&D programme [2] has been launched to develop large area HPD's adequate for the LHCb requirements. In addition to the high active area fraction, sensitivity in both the visible and the UV range, as well as fast LHC speed readout electronics are key requirements of the photodetectors.

In parallel to the project described in this article, an alternative $80 \mathrm{~mm}$ diameter cross-focused HPD design is being developed by another group in the LHCb collaboration [3] together with an industrial partner. As a back-up photodetector solution the commercially available multi-anode photomultiplier tubes R5900-64 by Hamamatsu are under investigation [4, 5]. 


\section{The 2048-pad, 5-inch HPD}

The HPD's, as shown in Fig. 2, consist of a cylindrical vacuum glass envelope of $127 \mathrm{~mm}$ diameter with a spherical entrance window made of borosilicate glass. A visible light transmittive bialkali photocathode $\left(\mathrm{K}_{2} \mathrm{CsSb}\right)$ is vacuum evaporated on the inside surface of the window. The photoelectrons are accelerated by a potential difference of the order of $20 \mathrm{kV}$ onto a silicon sensor of $50 \mathrm{~mm}$ diameter. Focusing electrodes, fixed in the glass envelope, demagnify the image by a factor 2.7. The demagnification is achieved by

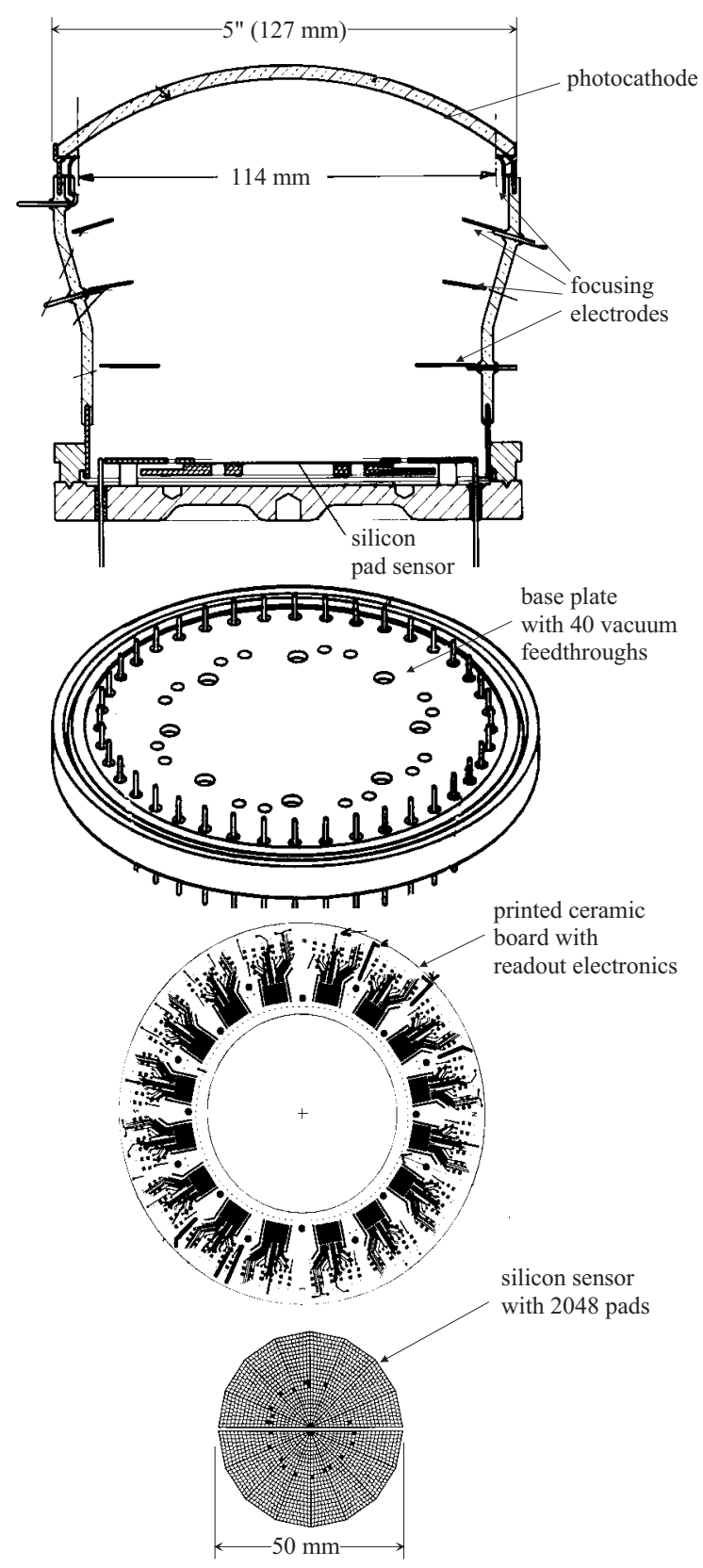

Figure 2: The upper figure shows a cross section of the 5-Inch HPD. Below the the base plate, the ceramic printed board and the silicon sensor are depicted. 
a fountain like electrostatic configuration. It is defined by a set of four focusing ring electrodes, including a bleeder electrode near the photocathode. At the bottom of the vacuum tube a stainless-steel flange is welded which incorporates a knife-edge. It fits into the $\mathrm{V}$-shaped groove in the base plate which is filled with de-gassed indium metal. A vacuum tight seal is made by applying a force of $25 \mathrm{kN}$.

The silicon sensor is glued to a 4-layer ceramic printed circuit board. The readout chips are placed at the sensor circumference. The ceramic board is mounted on the stainless-steel base plate which contains 40 vacuum feedthroughs to extract the electronic signals.

\subsection{The silicon sensor and the readout electronics}

Silicon sensors of $27.5 \mathrm{~mm}$ radius and $180 \mu \mathrm{m}$ thickness consisting of 16 sectors with in total 2048 pads have been produced. The inner $25 \mathrm{~mm}$ contain the pads (each $\approx 1 \times 1 \mathrm{~mm}^{2}$ ) and the outer $2.5 \mathrm{~mm}$ region receives the fanin traces from the pads for wire-bonding to the readout chip. This sensor has been successfully tested in vacuum with photoelectrons up to $20 \mathrm{keV}$ using 16 VA3 [6] analogue readout chips (128 channels: pre-amplifier, shaper, sample \& hold and multiplexer). The single electron signal/noise is measured to be $10 \sigma$ (where $\sigma$ is the Gaussian width of the pedestal), less than $18 \sigma$ measured for the previous 256 pad sensor [6], due to the increased noise level from the 16 readout chips and incomplete signal collection due to differences in the manufacturing of these sensors.

The VA3 chip has been used in the first stages of the R\&D effort because it has low noise, is tested and reliable. However, with 1-2 $\mu$ s peaking time, it is too slow for operation at LHC. The SCT128A [7] is a 25 ns peaking time, low noise, radiation-hard, 128 channel chip with a 128 element analogue pipeline originally developed for the ATLAS silicon tracker but seems well suited for use in the pad HPD. First SCT128A chips optimized for the readout of low capacity pad devices have now become available for laboratory tests. The measured figures for noise $\left(\approx 500 \mathrm{e}^{-} \mathrm{RMS}\right)$ and peaking time $(\leq 25 \mathrm{~ns})$ fully confirm the expectations.

\subsection{The electron optics}

Simulations with finite element codes (SIMION [8],MAFIA) predict an oneto-one mapping of photoelectrons out to $90 \%$ of the total tube diameter. As shown in Fig. 3 an uniform field geometry at the edge of the photocathode requires a fourth electrode, the so-called bleeder electrode. The potential of the bleeder has to be only a few hundred volts below the cathode potential.

In order to verify the electrostatic simulations of the HPD a special nonsealed version with a CsI photocathode has been produced. This UV sensitive cathode can be fabricated by a comparably simple evaporation process and even tolerates short exposures to air, which facilitates the handling of the components. 


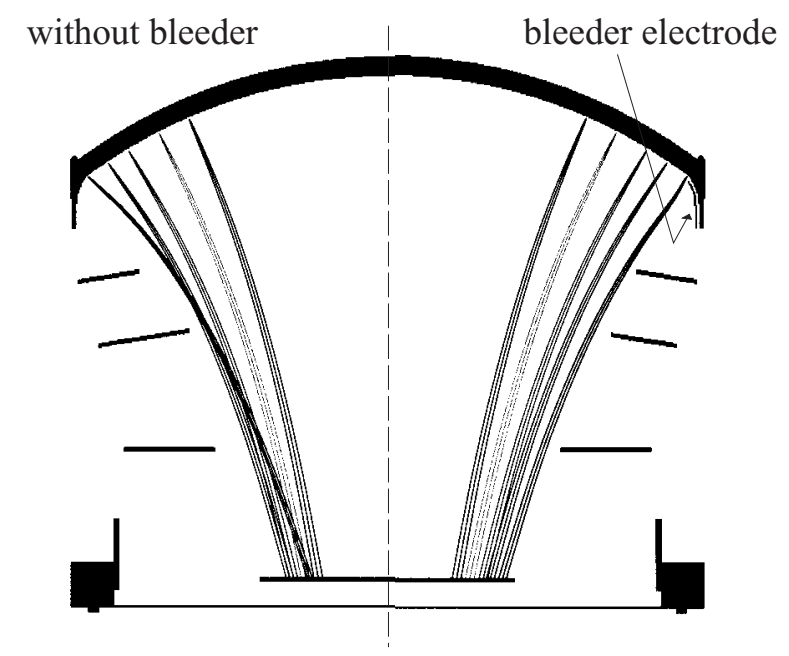

Figure 3: Simulation of electron trajectories with the code SIMION. The electrons are emitted from the cathode at a fixed energy of $1 \mathrm{eV}$ and at angles $\theta=-45^{\circ}, 0^{\circ}$ and $45^{\circ}$ to the normal of the window. Without bleeder electrode (left part) the trajectories cross over leading to ambiguities in the mapping. The bleeder electrode (right part) restores the field distortions and provides an unique mapping.

In this case the entrance window consists of fused quartz and has a wavelength cut-off at about $160 \mathrm{~nm}$. The vacuum envelope and the fully equipped base plate were mounted in a vacuum tank. By means of a moveable pulsed UV light source with a narrow beam spot the mapping of the photocathode

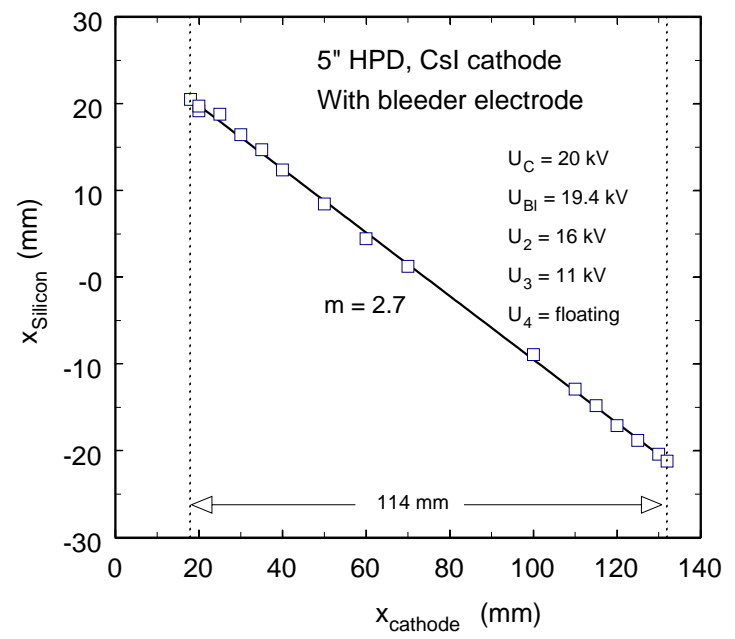

Figure 4: Results of the electrostatic tests. The figure shows the position of the photoelectrons hitting the silicon sensor versus the position of the photons on the photocathode. 


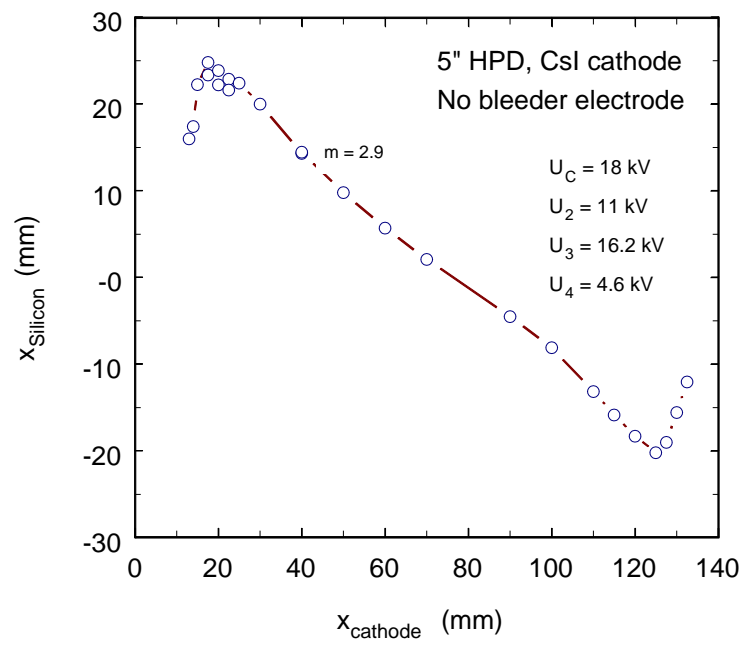

Figure 5: Ambiguous mapping without bleeder electrode. A linear demagnification can be achieved only in the central part of the tube.

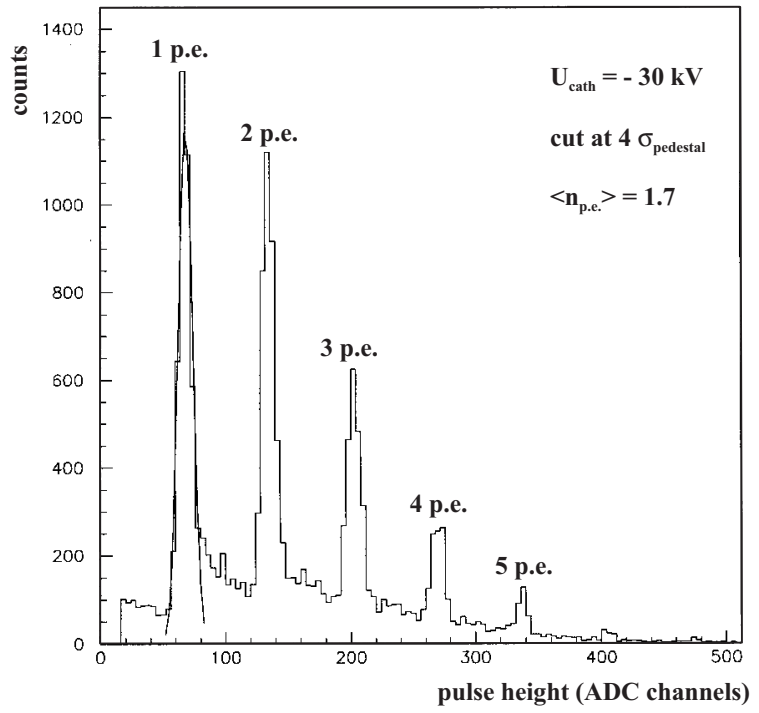

Figure 6: Pulseheight spectrum observed on a single pad at a cathode potential of $-30 \mathrm{kV}$. The pedestal is suppressed by a $4 \sigma$ cut. The signal to noise ratio is close to 12 .

surface on the silicon sensor could be precisely determined. The result is shown in Fig. 4. The slope of the curve gives the demagnification of the HPD, which can be adjusted over a wide range by varying the potentials on the focusing electrodes. The set of potentials shown in the figure results in a linear demagnification of 2.7 over the full geometrically accepted diameter of $114 \mathrm{~mm}$. In a further test a tube without bleeder electrode was used. As predicted by the simulations ambiguities at large radial distances from the tube axis have been found (Fig. 5).

In these tests the tube was stably operated at voltages up to $30 \mathrm{kV}$. 


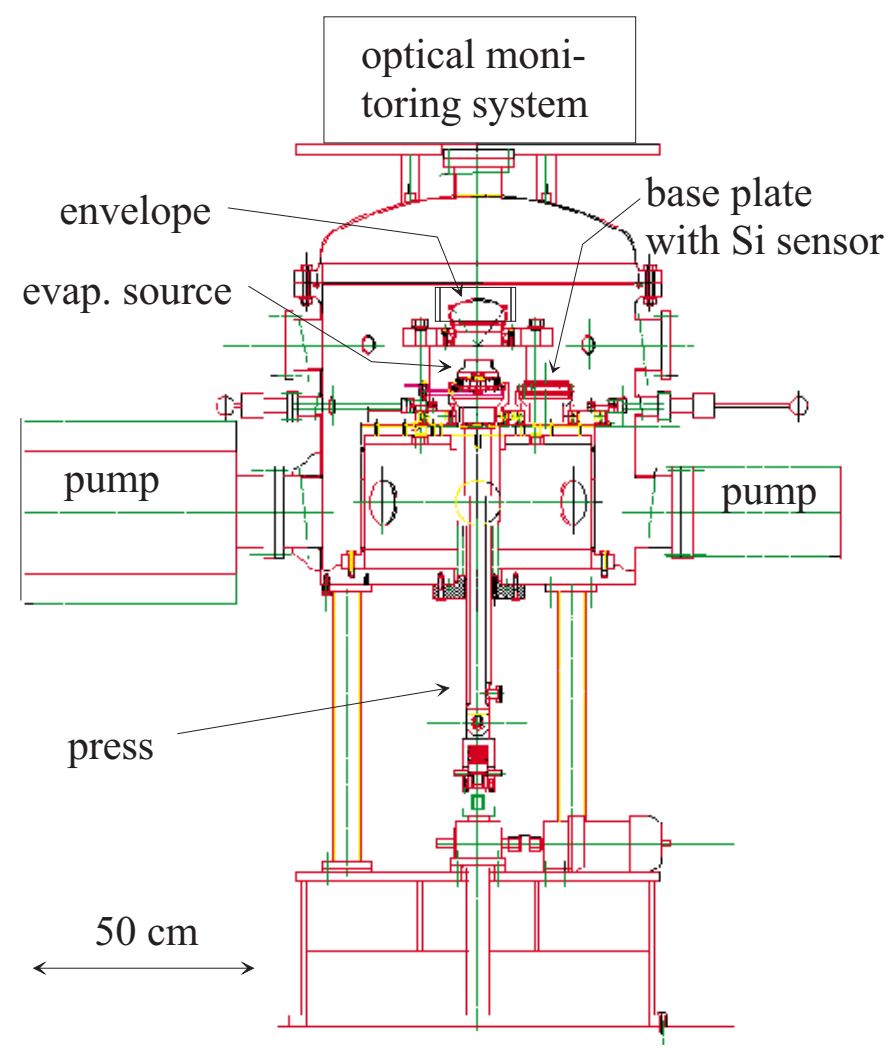

Figure 7: Side view of the photocathode deposition apparatus.

A pulsehight spectrum, observed on a single pad at a cathode potential of $30 \mathrm{kV}$ is shown in Fig. 6. The pedestal peak has been suppressed by applying a $4 \sigma$ cut, where $\sigma$ is the Gaussian width of the pedestal. The average number of photons per lamp pulse is 1.7 .

\section{HPD fabrication}

\subsection{The evaporation plant}

The fabrication of the HPD requires the vacuum evaporation of the bialkali photocathode and the subsequent encapsulation of the tube with the base plate. In order to minimise the risk of contaminating the silicon detector during the photocathode processing and the sealing an external deposition process combined with a cold indium joint technique has been developed. The photocathode is deposited onto the inside window through the bottom hole of the tube. The tube is afterwards sealed off with the base plate on which the silicon detector and the readout electronics are mounted.

An evaporation facility [9], as illustrated in Figure 7, consisting of a large cylindrical vacuum tank with a removable lid has been built. Four movable carriages, which are activated via linear motion vacuum feedthroughs, can be moved under the HPD glass envelope which is held by a rigid support structure in the centre of the vacuum tank. Two of the carriages support the 

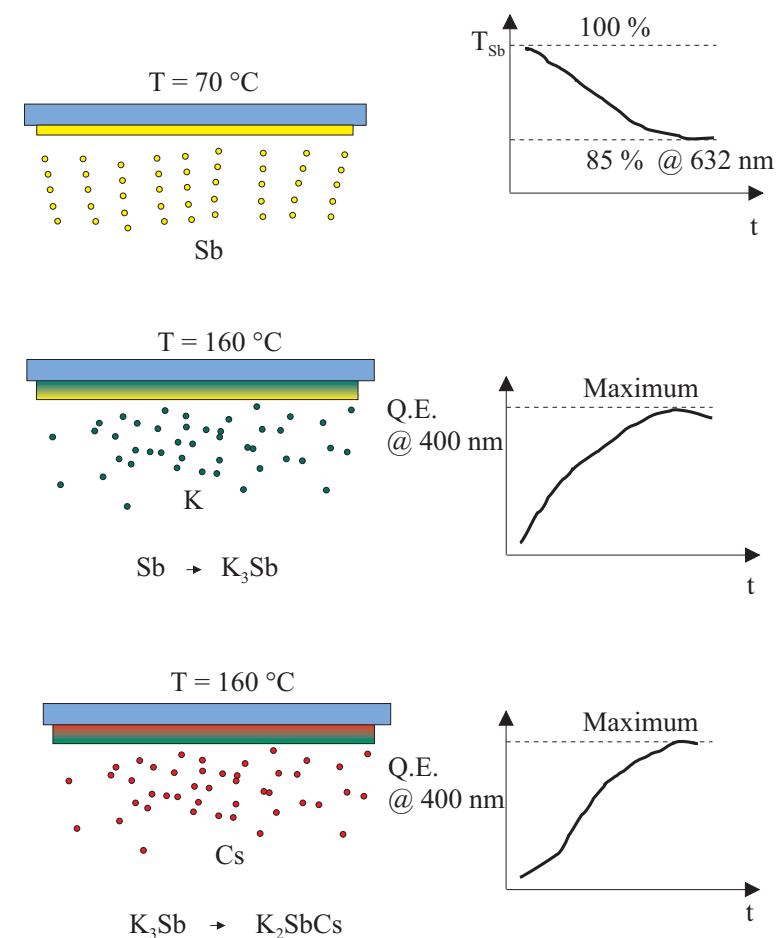

Figure 8: Schematic representation of the bialkali photocathode process.

evaporation sources ( $\mathrm{Sb}, \mathrm{K}, \mathrm{Cs}$ ), the third one is loaded with the HPD base plate. The fourth carriage is equipped with a glow discharge electrode which allows to clean the window surface prior to evaporation by means of electron and ion bombardment in a plasma discharge. The facility is equipped with a powerful pumping system and is baked out at temperatures around $250^{\circ} \mathrm{C}$. Vacuum levels below $\left(1 \times 10^{-7} \mathrm{~Pa}\right)$ are reached. The vacuum quality which is essential for the stability of the photocathode is monitored by means of a residual gas analyser. A cylindrical heating element allows to precisely control the temperature of the vacuum envelope during the deposition process. An optical system monitors the transmission of the evaporated films. The quantum efficiency of the photocathode is measured online by means of a calibrated light source. Interference filters allow to measure the efficiency as a function of the wavelength. A PC based distributed slow control system monitors the essential process parameters and controls various process parameters including the substrate temperature and the degassing of the evaporation sources. Automation of most of the process steps is foreseen at a later stage.

\subsection{The bialkali photocathode process}

Our standard photocathode fabrication process which is schematically shown in Figure 8 starts with the deposition of a Antimony film. The deposition is stopped when the transmission of the film for red light $(\lambda=632 \mathrm{~nm})$ is reduced to $85 \%$. During the $\mathrm{Sb}$ deposition the substrate, i.e. the window is 
kept at a constant temperature of $70^{\circ} \mathrm{C}$. The window is then heated to $160^{\circ} \mathrm{C}$ where first Potassium and subsequently Cesium is evaporated. In both cases the evaporation is continued until a maximum of the measured quantum efficiency at $\lambda=400 \mathrm{~nm}$ is observed. Subsequent alternate K and Cs evaporation may lead to a further increase of the efficiency and compensates for the re-evaporation losses from the cathode during the slow temperature ramp down. Figure 9 shows the quantum efficiency of some bialkali photocathodes recently produced. They reach efficiencies close to the reference values taken from a Hamamatsu photomultiplier catalog. Although a good fraction of the cathodes shown in Fig. 10 has not been produced under optimised conditions, the wide spread of results underlines the difficulty of the bialkali process. The final result depends also on a number of parameters like temperature gradients, evaporation rates and substrate surface quality which are sometimes difficult to quantify and to control.

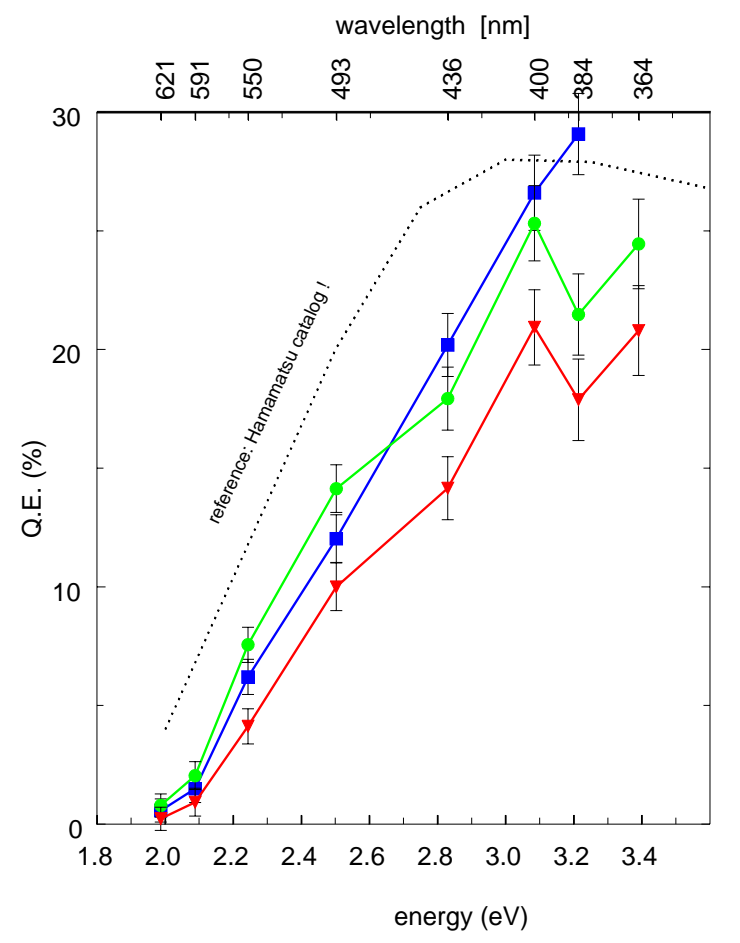

Figure 9: Quantum efficiency of bialkali photocathodes.

A light scan of the cathode surface reveals in general good uniformity of the detection efficiency. An efficiency drop of typically $20 \%$ is observed close to the edge of the window.

\subsection{The cold Indium sealing technique}

A cold Indium sealing technique has been developed which avoids any thermal load and contamination of both the photocathode and the Silicon sensor. Since the lifetime of a HPD has to be of the order of ten years and the pressure inside the envelope must not exceed about $10^{-5} \mathrm{~Pa}$, leak and outgassing 


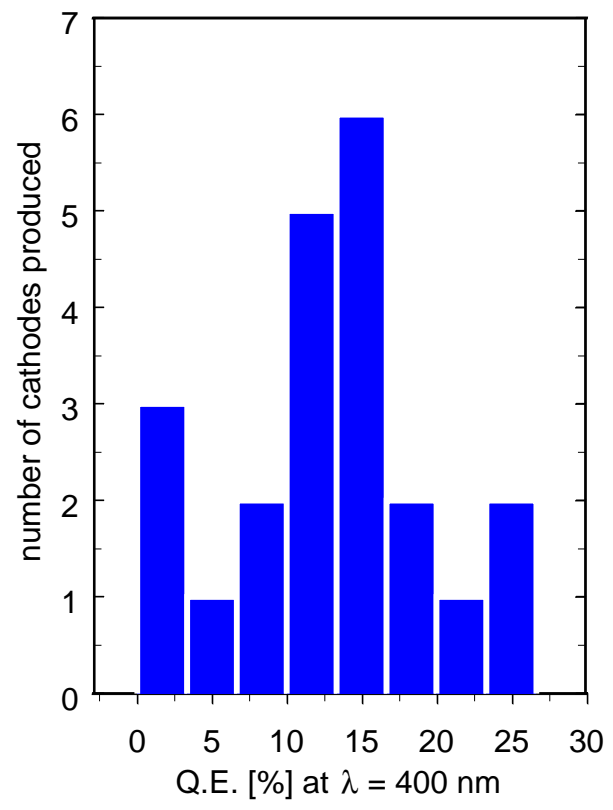

Figure 10: Distribution of the quantum efficiency measured at $\lambda=400 \mathrm{~nm}$ of 22 photocathodes.

rates have to be kept on extremely low levels. To improve the wetability of Indium on the stainless steel surfaces, both the knife-edge and the groove is galvanically deposited with Nickel and Indium films of about $2 \mu \mathrm{m}$ and $10 \mu \mathrm{m}$ thickness, respectively. A high purity Indium wire (99.999\%) is premelted and degassed in the groove at temperatures around $400^{\circ} \mathrm{C}$ resulting in an uniform surface. After the photocathode processing the vacuum seal is made at temperatures well below $100^{\circ} \mathrm{C}$ ("cold") by pushing the base plate against the steel flange of the envelope by means of an accurately controllable mechanical press. The applied force of $25 \mathrm{kN}$ corresponds to about $60 \mathrm{~N}$ per $\mathrm{mm}$ joint length. Since the Indium metal has been fully degassed before, no gas is released during the sealing process.

In a separate set-up a stainless-steel cell, consisting of two disks with the same knife-edge and groove geometry, has been sealed. Repeated Helium leak tests with a sensitivity of $10^{-8} \mathrm{~Pa} \cdot l \cdot \mathrm{s}^{-1}$ over a period of several months have not revealed any indication of a leak.

A phototube in which the silicon sensor is replaced by a simple metallic collection anode has recently been sealed. Fig. 11 shows the variation of the photocurrent of this tube measured at constant illumination over a period of 80 hours. Apart from small periodic fluctuations, which might be due to temperature variations in the laboratory, no degradation of the cathode is observed.

To further improve the vacuum quality inside the tube, the integration of a non-evaporable getter strip is foreseen at a later stage. 


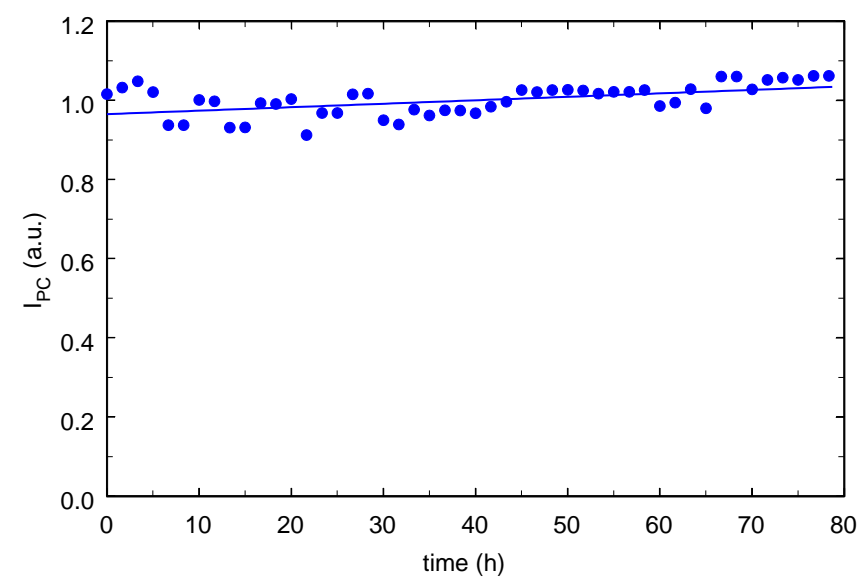

Figure 11: Variation of the photocurrent of a sealed phototube over an 80 hours period of constant illumination.

\section{Summary and outlook}

All components to fabricate large area HPD's (silicon sensor, electronics, glass envelope) have been developed and successfully tested. Two glass envelopes with fused quartz windows have been coated with CsI transmittive photocathodes. One has been used to study the focusing properties of the electrode structure whereas the second will be used to produce a sealed UV sensitive CsI 2048-pad HPD. An effective acceptance of 114 out of $127 \mathrm{~mm}$ has been demonstrated with a demagnification factor $m \approx 2.7$.

The new evaporation plant was commissioned with success and allows to produce visible light $\mathrm{K}_{2} \mathrm{CsSb}$ photocathodes with high quantum efficiency. The further optimisation of the sensitivity and the stability of the photocathodes is subject of the current studies.

The cold indium sealing technique has proven to be compatible with the standard bialkali photocathode process and provides excellent vacuum tightness. The fabrication of a sealed visible light HPD with the silicon sensor incorporated in the tube is expected in the nearest future. Beam tests of both a CsI and bialkali HPD with Cherenkov radiators are scheduled for the end of 1998.

\section{Acknowledgements}

We wish to thank M. Alemi (CERN EP/TA2) and D. Ferenc (Univ. Regensburg, Germany) for the electrostatic simulations of the HPD. We want to express our gratitude to M. Brouet (CERN LHC/VAC) for many helpful discussions on the secrets of ultrahigh vacuum systems and for the very efficient support by his team of technicians. We are indebted to S. Mathot and A. Lasserre (CERN EST/MS) for valuable hints and very competent electroplating of the grooves and knife edge surfaces. The continuous support of the project by P. Giusti (INFN Bologna, Italy), F. Garibaldi (INFN Rome, 
Italy), and E. Nappi (Univ. of Bari, Italy) is gratefully acknowledged. Part of this work was supported by the CERN-LAA project.

\section{References}

[1] LHCb Technical Proposal. CERN/LHCC 98-4, LHCC/P4

[2] E. Chesi et al., $L H C-B / 96-010$

[3] M. Campbell et al., LHCb 98-035

M. Alemi et al., contribution to this conference.

[4] A. Duane et al., LHCb 98-39

[5] R. Forty, LHCb 98-38

[6] P. Weilhammer et al., Nucl. Instr. Meth. A383 (1996) 89

[7] S. Anghinolfi et al., IEEE Trans. Nucl. Sci. 44 (1997) 298

[8] D. Ferenc, Proc. of Erice workshop on new detctors, Nov. 1997, World scientific, in press.

[9] A. Braem et al., $\mathrm{LHCb}$ 98-07 